\title{
ON THE MULTIPLICITIES OF EIGENVALUES OF A HERMITIAN MATRIX WHOSE GRAPH IS A TREE
}

\author{
C.M. DA FONSECA
}

\begin{abstract}
A different approach is given to recent results due mainly to R.C. Johnson and A. Leal Duarte on the multiplicities of eigenvalues of a Hermitian matrix whose graph is a tree. The technics developed are based on some results of matchings polynomials and use a work by O.L. Heilmann and E.H. Lieb on an apparently unrelated topic.
\end{abstract}

KEYWORDS: eigenvalues, graph, tree, symmetric matrices, vertex degrees. AMS SubJeCt Classification (2000): 15A18, 15A57, 05C50, 05C05, $05 \mathrm{C} 12$.

\section{Introduction}

In recent years, the interest of study of the multiplicities of eigenvalues of Hermitian matrices whose graph is a given tree has grown substantially and it has been led by C.R. Johnson, A. Leal Duarte and others (cf. [13, 14, 15, 16, 17, 18]) inspired by the work of J. Genin and J.S. Maybee [6] and S. Parter [21]. For 01-adjacency matrices several results are known, but for more general ones few are.

In 1999, Johnson and Leal Duarte [13] studied the maximum multiplicity of an eigenvalue of matrix whose graph is a given tree. They considered some inequalities between different parameters associated with the tree and then they expressed the result in terms of them. Later they obtained an inequality between the minimum number of distinct eigenvalues of a Hermitian matrix whose graph is a tree $T$ and the number of vertices in a longest path in $\mathrm{T}$ (cf. [14]).

One of the concepts explored by Johnson, Leal Duarte and Saiago [13, 17] is the minimum number of vertex disjoint paths, occurring as induced subgraphs of a tree $T$, which cover all the vertices of $T$. This concept had already been studied by O. Ore [20], F.T. Boesch, S. Chen and J.A.M. McHugh [1] and by P.J. Slater [22]. Using essentially some constructions due to Slater, in the next section we can give a shorter proof of a Johnson and Leal Duarte's result.

Received April 15, 2004.

This work was supported by CMUC - Centro de Matemática da Universidade de Coimbra. 
In a work elaborated apparently in a distinct context, motivated by problems in quantum chemistry, O.L. Heilmann and E.H. Lieb [11] established crucial results which are in the base of some recent developments on the matchings polynomial (cf. $[7,8,9,19]$ ). In fact, there are close relations between the theory of matchings in graphs and the characteristic polynomial of adjacency matrix of a graph. Recall that a $k$-matching in a graph is a set of $k$ disjoint edges, no two of which have a vertex in common. Denoting $p(G, k)$ as the number of $k$-matchings in a graph $G$, we define

$$
\mu(G, x):=\sum_{k}(-1)^{k} p(G, k) x^{n-2 k}
$$

the matchings polynomial of $G$. Though the matchings polynomial of a graph has many interesting properties, the task of computing this polynomial for a given graph is complex. In general there is no easy way of computing $\mu(G, x)$. Thus, the matchings polynomial is in this regard a more intractable object than the characteristic polynomial. Nonetheless, it is known that $G$ is a forest if and only if both polynomials coincide and there also simple recurrences that enable us to compute the matchings polynomials of small graphs with some facility. For example, the matchings polynomials of bipartite graphs are essentially the same as "rook polynomials" (cf. [9]). An unexpected property of the matchings polynomials is that all zeros are real. In the paper [11] we can find three distinct proofs of this fact. Therefore if $G$ is a tree, then all the eigenvalues of the (ordinary) adjacency matrix of $G$ are real.

Basing our approach solely on Heilmann and Lieb's result we explore some properties of the characteristic polynomials of a general matrix whose graph is a given tree and which facilitates simpler proofs of the main results of $[13,14]$. We also establish some results on the multiplicities of the eigenvalues of a tree.

\section{Path coverings}

A graph $G$ is a pair $(V, E)$, with vertex set $V=V(G)=\{1, \ldots, n\}$, and where $E=E(G)$, the edge set, is a subset of the direct product $V \times V$. We say that the vertices $i$ and $j$ are adjacent, and write $i \sim j$, if $(i, j)$ is an edge of $G$, with $i \neq j$. The symbol $\simeq$ means adjacent or equal.

If $S$ is a subset of $V(G)$, then $G \backslash S$ is the subgraph of $G$ induced by the vertices not in $S$. In particular, if $u \in V(G)$, then $G \backslash u$ is the graph obtained by removing $u$ and all its attendant edges. 
A tree is a connect graph without cycles and a forest is a graph in each component is a tree. In this paper we consider finite graphs possibly with loops (i.e., $(i, i)$ may be an edge). If to each edge $(i, j)$ is assigned a complex number, we have a weighted graph. We shall focus our attention on trees.

Given a tree $T$, we define the path-covering number, denoted by $\zeta(T)$, as the smallest number of vertex disjoint paths of $T$ that cover all the vertices of $T$.

Historically, O. Ore [20] first introduced the graphical invariant $\zeta$ followed by others (e.g. $[1,22])$. Ore defined the vertex disjoint path coverings of the vertices of a graph in such way the paths contain a maximum number of edges, which is equivalent tho the definition of $\zeta$. Later, Farrell [4] established an algorithm for finding the number of ways of doing it. In [22], Slater gave a procedure for forming the smallest referred collection of disjoint paths.

Let us recall some definitions for a tree $T$. If $v$ is a vertex of $T$, a branch of $T$ at $v$ is the subgraph induced by $v$ and one of the components of $T \backslash v$. If $\operatorname{deg}(v) \geq 3$, we call branch path at $v$ to a branch of $T$ at $v$ which is a path. In this conditions the vertex $v$ is the stem of the branch path at $v$, and the subgraph of $T$ consisting of $v$ and all its branch paths will be called a leaf with stem $v$. Note that distinct leaves of $T$ must be disjoint. An end leaf is a leaf whose stem has $\operatorname{deg}(v)$ or $\operatorname{deg}(v)-1$ branch paths.

Denote $D(T)$ as the number of vertices in $T$ of degree at least three.

Lemma 2.1. Suppose that the tree $T$ is not a path. If $D(T)=1$, then there is exactly one (end) leaf. Otherwise, there are at least two end leaves whose stems, $v_{1}$ and $v_{2}$, have $\operatorname{deg}\left(v_{1}\right)-1$ and $\operatorname{deg}\left(v_{2}\right)-1$ branch paths, respectively.

The next lemma establishes an algorithm for determining $\zeta(T)$.

Lemma $2.2([22])$. Let $T$ be a tree. If $D(T)=0$, then $\zeta(T)=1$. If $D=1$, let $v$ be the vertex of degree at least three, then $\zeta(T)=\operatorname{deg}(v)-1$. Otherwise, let $L$ be an end leaf with stem $v$. Then $\zeta(T)=\zeta(T \backslash L)+\operatorname{deg}(v)-2$.

One of the parameters defined in [13] is $\Delta(T)=\max \{p-q \mid$ there exist $q$ vertices of $T$ whose deletions leaves $p$ paths $\}$

Considering the stems of the Lemma 2.2, we may state

Corollary 2.3 ([13]).

$$
\zeta(T)=\Delta(T) .
$$


According to Lemma 2.2, note that the value of $\Delta(T)=\zeta(T)$ depends on some of the vertices of $T$ of degree greater or equal to 3 . Johnson and Saiago [17] gave tight bounds for $\Delta(T)$ in terms of all vertex whose degrees are greater than 2 . This result also comes out as a corollary of the Lemma 2.2 .

Corollary 2.4 ([17]). Denote by $\epsilon$ the number of edges adjacent to two vertices of degree greater than $3, v_{1}, \ldots, v_{k}$. Then

$$
1+\sum_{j=1}^{k}\left(\operatorname{deg}\left(v_{j}\right)-2\right)-\epsilon \leq \Delta(T) \leq 1+\sum_{j=1}^{k}\left(\operatorname{deg}\left(v_{j}\right)-2\right) .
$$

\section{The characteristic polynomial of weighted trees}

If $A=\left(a_{i j}\right)$ is a Hermitian matrix, the (weighted) graph of $A, G=G(A)$, is determined entirely by the off-diagonal entries of $A$ : the vertex set of $G(A)$ is $\{1, \ldots, n\}$ and $i$ and $j$ are adjacent if and only if $a_{i j} \neq 0$. If $A$ is a 01-matrix, with main diagonal equal to zero, then $A$ is the adjacency matrix of $G(A)$. On the other hand, given a graph $G$, we define

$$
\mathcal{H}(G)=\left\{A \mid A=A^{*}, G(A)=G\right\},
$$

the set of all Hermitian matrices that share a common graph $G$.

If $e=(i, j)$ is an edge of $G$, then $G \backslash e$ is obtained by deleting $e$ but not the vertices $i$ or $j$. In this case the matrix of $G \backslash e$ is equal to the one of $G$, except the $(i, j)$-entry and, by symmetry, the $(j, i)$-entry, which are zero. Finally, $\varphi(G, \lambda)$, or simply $\varphi(G)$, is the characteristic polynomial of $A(G)$, i.e., $\varphi(G, \lambda)=\operatorname{det}(\lambda I-A(G))$, sometimes referred as the characteristic polynomial of $G$.

Lemma 3.1. Let $F$ be a forest with components $T_{1}, \ldots, T_{\ell}$, then $\varphi(F)=$ $\varphi\left(T_{1}\right) \cdots \varphi\left(T_{\ell}\right)$.

Let us define $w_{i j}(A)=\left|a_{i j}\right|^{2}$ if $i \neq j$ and, otherwise, $w_{i i}(A)=a_{i i}$. Sometimes we abbreviate to $w_{i j}$. The next result provides a general recurrence relation between different characteristic polynomials.

Lemma 3.2. If $e=(i, j)$ is an edge in a (weighted) tree $T$, then

$$
\varphi(T, \lambda)=\varphi(T \backslash e, \lambda)-w_{i j} \varphi(T \backslash i j, \lambda) .
$$


Proof: Let $E_{i j}$ be the matrix with $i j$-entry equal to 1 , and all other entries equal to zero. Denote by $E$ the sum $a_{i j} E_{i j}+\bar{a}_{i j} E_{j i}$. Notice that

$$
A(T)=A(T \backslash e)+E .
$$

Since the determinant is a multilinear function on the columns and $T$ is a tree, we get (3.1).

Theorem 3.3. Let $i$ be a vertex of a weighted tree $T$. Then

$$
\varphi(T, \lambda)=\left(\lambda-w_{i i}\right) \varphi(T \backslash i, \lambda)-\sum_{k \sim i} w_{k i} \varphi(T \backslash k i, \lambda) .
$$

Proof: The equality (3.2) can be derived by iterating formula (3.1).

A routine induction argument, based on (3.2), gives us an expression for the derivative of characteristic polynomial.

Corollary 3.4. If $T$ is a (weighted) tree, then

$$
\varphi^{\prime}(T, \lambda)=\sum_{i \in V(T)} \varphi(T \backslash i, \lambda) .
$$

From Theorem 3.3 we can get the expression for the determinant of a tree.

Corollary 3.5. If $T$ is a weighted tree and $i \in V(T)$, then

$$
\operatorname{det}(T)=\sum_{j \simeq i}(-1)^{\delta_{i, j}} w_{i j} \operatorname{det}(T \backslash i j),
$$

where $\delta_{i, j}$ is the Kronecker symbol.

We can also establish an analogous result for the permanent.

Corollary 3.6. If $T$ is a weighted tree and $i \in V(T)$, then

$$
\operatorname{per}(T)=\sum_{j \simeq i} w_{i j} \operatorname{det}(T \backslash i j) .
$$

We now state a result which we will keep in mind throughout. The general interlacing theorem between the eigenvalues of a Hermitian matrix and one principal submatrix is well known in the literature (see e.g. [12]).

Theorem 3.7. Let $T$ be a tree on $n$ vertices and $A \in \mathcal{H}(T)$. Then all eigenvalues of $A(T)$ are real, say

$$
\lambda_{1} \leq \lambda_{2} \leq \cdots \leq \lambda_{n}
$$


Furthermore, if $i$ is a vertex in $T$ and $\mu_{1} \leq \mu_{2} \leq \cdots \leq \mu_{n-1}$ are the eigenvalues of $A(T \backslash i)$, then

$$
\lambda_{1} \leq \mu_{1} \leq \lambda_{2} \leq \mu_{2} \leq \cdots \leq \mu_{n-1} \leq \lambda_{n},
$$

i.e., the eigenvalues of $A(T)$ interlace those of $A(T \backslash i)$.

This theorem has a well known corollary for tridiagonal matrices already proved elsewhere.

Corollary 3.8. Let $P$ be a path on $n$ vertices and $A \in \mathcal{H}(P)$. Then $A(P)$ has $n$ distinct real eigenvalues.

\section{Christoffel-Darboux identity}

As we pointed out before, the work of Heilmann and Lieb [11] has had some important implications on the theory of matchings polynomials. Here we derive some important identities for matrices of the set $\mathcal{H}(T)$.

One of the most important tools in the study of orthogonal polynomials is the Spectral Theorem for orthogonal polynomials, which states that any (monic) orthogonal polynomial sequence $\left\{p_{n}\right\}_{n \geq 0}$ is characterized by a threeterm recurrence relation

$$
p_{n+1}(x)=\left(x-\beta_{n}\right) p_{n}(x)+\gamma_{n} p_{n-1}(x), \quad n=0,1,2, \ldots
$$

with initial conditions $p_{-1}(x)=0$ and $p_{0}(x)=1$, where $\left\{\beta_{n}\right\}_{n \geq 0}$ and $\left\{\gamma_{n}\right\}_{n \geq 0}$ are sequences of complex numbers such that $\gamma_{n+1}>0$ for all $n=0,1,2, \ldots$ (for more details see e.g. [2]). We can explore the existing similarities between the recurrence relations (3.2) and (4.1) and get some Christoffel-Darboux type formula for special polynomials.

Let $P_{i j}$ denote the (unique) path in a tree joining vertex $i$ to $j$ and. Given a path $\mathrm{P}$ in $T$ with more than one vertex, let us define $W(P)=\prod_{(i, j)} w_{i j}(P)$, where the product is taken over the edges $(i, j)$ of $P$, with $i<j$.

Theorem 4.1 (Christoffel-Darboux Identity). Let $T$ be a weighted tree on $n$ vertices. For every vertex $i \in V(T)$,

$\varphi(T, \lambda) \varphi(T \backslash i, \mu)-\varphi(T, \mu) \varphi(T \backslash i, \lambda)=(\lambda-\mu) \sum_{j=1}^{n} W\left(P_{i j}\right) \varphi\left(T \backslash P_{i j}, \lambda\right) \varphi\left(T \backslash P_{i j}, \mu\right)$.

Proof: For trees with one or two vertices the result is trivial. From (3.2), we have the equations 


$$
\lambda \varphi(T \backslash i, \lambda) \varphi(T \backslash i, \mu)=\varphi(T, \lambda) \varphi(T \backslash i, \mu)+\sum_{k \simeq i} w_{k i} \varphi(T \backslash k i, \lambda) \varphi(T \backslash i, \mu)
$$

and

$$
\mu \varphi(T \backslash i, \mu) \varphi(T \backslash i, \lambda)=\varphi(T, \mu) \varphi(T \backslash i, \lambda)+\sum_{k \simeq i} w_{k i} \varphi(T \backslash k i, \mu) \varphi(T \backslash i, \lambda) .
$$

Subtracting the second equation from the first we get

$$
\begin{array}{r}
(\lambda-\mu) \varphi(T \backslash i, \mu) \varphi(T \backslash i, \lambda)=\varphi(T, \lambda) \varphi(T \backslash i, \mu)-\varphi(T, \mu) \varphi(T \backslash i, \lambda) \\
-\sum_{k \sim i} w_{k i}[\varphi(T \backslash i, \lambda) \varphi(T \backslash k i, \mu)-\varphi(T \backslash i, \mu) \varphi(T \backslash k i, \lambda)] .
\end{array}
$$

Applying the hypothesis on $\varphi(T \backslash i, \lambda) \varphi(T \backslash k i, \mu)-\varphi(T \backslash i, \mu) \varphi(T \backslash k i, \lambda)$, with the convention $\varphi(\emptyset, \lambda)=1$, we get the result.

Corollary 4.2. Let $T$ be a weighted tree on $n$ vertices. For every vertex $i \in V(T)$,

$$
\varphi^{\prime}(T, \lambda) \varphi(T \backslash i, \lambda)-\varphi(T, \lambda) \varphi^{\prime}(T \backslash i, \lambda)=\sum_{j=1}^{n} W\left(P_{i j}\right) \varphi\left(T \backslash P_{i j}, \lambda\right)^{2} .
$$

Proof: Letting $\mu \rightarrow \lambda$ in (4.2), we get (4.3) since we may write

$$
\begin{aligned}
& \varphi(T, \lambda) \varphi(T \backslash i, \mu)-\varphi(T, \mu) \varphi(T \backslash i, \lambda)= \\
& \quad=[\varphi(T, \lambda)-\varphi(T, \mu)] \varphi(T \backslash i, \mu)-[\varphi(T \backslash i, \lambda)-\varphi(T \backslash i, \mu)] \varphi(T, \lambda) .
\end{aligned}
$$

If we consider the sum over all vertices of $T$ in (4.3), from the Corollary 3.4 we get:

\section{Corollary 4.3.}

$$
\varphi^{\prime}(T, \lambda)^{2}-\varphi^{\prime \prime}(T, \lambda) \varphi(T, \lambda)=\sum_{i, j=1}^{n} W\left(P_{i j}\right) \varphi\left(T \backslash P_{i j}, \lambda\right)^{2} .
$$

Theorem 4.4. Let $T$ be a weighted tree on $n$ vertices. For every distinct vertices $i, j \in V(T)$,

$$
\varphi(T \backslash i, \lambda) \varphi(T \backslash j, \lambda)-\varphi(T \backslash i j, \lambda) \varphi(T, \lambda)=W\left(P_{i j}\right) \varphi\left(T \backslash P_{i j}, \lambda\right)^{2} .
$$


Proof: Once again the cases $n=1$ and $n=2$ are trivial. We use induction on $n$ in an analogous way of the propositions before. For that we only have to consider (3.2) and

$$
\varphi(T \backslash j, \lambda)=\left(\lambda-w_{i i}\right) \varphi(T \backslash i j, \lambda)-\sum_{k \sim i} w_{k i} \varphi(T \backslash k i j, \lambda) .
$$

\section{Multiplicities of eigenvalues}

For any square matrix $A, m_{A}(\theta)$ denotes the (algebraic) multiplicity of $\theta$ as an eigenvalue of $A$; if $\theta$ is not an eigenvalue of $A$, then we will write $m_{A}(\theta)=0$. The Theorem 3.7 has a straightforward consequence for $A \in \mathcal{H}(T)$, where $T$ is a tree:

$$
\left|m_{A(T \backslash i)}(\theta)-m_{A(T)}(\theta)\right| \leq 1 \text {. }
$$

Suppose $m_{A(T)}(\theta)>0$, a vertex $i$ of $T$ is $\theta$-essential, $\theta$-neutral and $\theta$ positive if $m_{A(T \backslash i)}(\theta)=m_{A(T)}(\theta)-1, m_{A(T \backslash i)}(\theta)=m_{A(T)}(\theta)$ and $m_{A(T \backslash i)}(\theta)=$ $m_{A(T)}(\theta)+1$, respectively. If $\theta$ is understood, we shall omit the mention of $\theta$.

Note that each $A \in \mathcal{H}(T)$ has at least one essential vertex. Indeed, the multiplicity of $\theta$ as zero of $\varphi^{\prime}(T, \lambda)$ is $m_{A(T)}(\theta)-1$. If $m_{A(T \backslash i)}(\theta) \geq m_{A(T)}(\theta)$, for all vertices $i$ in $T$, then by Corollary 3.4 the multiplicity of $\theta$ as zero of $\varphi^{\prime}(T, \lambda)$ is at least $m_{A(T)}(\theta)$.

Theorem 5.1. Let $P$ a path in the tree $T$ and $A \in \mathcal{H}(T)$. If $\theta$ is an eigenvalue of $A(T)$, then

$$
m_{A(T \backslash P)}(\theta) \geq m_{A(T)}(\theta)-1 .
$$

Proof: Suppose that $\theta$ is an eigenvalue of $A(T)$ with $m_{A}(\theta)>1$. Then $\theta$ is a zero of $\varphi^{\prime}(T, \lambda)^{2}-\varphi^{\prime \prime}(T, \lambda) \varphi(T, \lambda)$ with multiplicity at least $2 m_{A}(\theta)-2$. From (4.4), $\theta$ is a zero of nonnegative summation $\sum_{i, j=1}^{n} W\left(P_{i j}\right) \varphi\left(T \backslash P_{i j}, \lambda\right)^{2}$, and therefore $\theta$ has multiplicity of each $\varphi\left(T \backslash P_{i j}, \lambda\right)$ at least $m_{A}(\theta)-1$.

In general, we say that a path $P$ is $P$-essential if $m_{A(T \backslash P)}(\theta)=m_{A(T)}(\theta)-1$. Given any $\theta$-essential vertex $i$, with $\theta \neq a_{i i}$, there is an adjacent vertex $j$, such that the path $i j$ is essential. For, if $m_{A(T \backslash i k)}(\theta) \geq m_{A(T)}(\theta)$, for all $k \sim i$, then by (3.2), $m_{A(T \backslash P)}(\theta) \geq m_{A(T)}(\theta)$, which is a contradiction.

We also point out that if a path $P_{i j}$ is essential, for some vertex $j$, then $i$ is an essential vertex. In fact, suppose that $m_{A(T \backslash i)}(\theta) \geq m_{A(T)}(\theta)$. For any $j(\neq i)$, the multiplicity of $\theta$ as a zero of $\varphi(T \backslash i, \lambda) \varphi(T \backslash j, \lambda)-\varphi(T \backslash i j, \lambda) \varphi(T, \lambda)$ 
is at least $2 m_{A(T)}(\theta)-1$. It is in fact, by $(4.5)$, at least $2 m_{A(T)}(\theta)$, and therefore $m_{A\left(T \backslash P_{i j}\right)}(\theta) \geq m_{A(T)}(\theta)$.

Given a tree $T$ on $n$ vertices, in [13], Johnson and Leal Duarte consider the maximum multiplicity of any single eigenvalue among all matrices in $\mathcal{H}(T), M(T)$. They established inequalities between the quantities $\zeta(T)$ and $\Delta(T)$ defined in the section $2, M(T)$ and $n-\min _{A \in \mathcal{H}(T)} \operatorname{rank} A$, and prove the following:

Corollary 5.2 ([13]). For each (weighted) tree T,

$$
M(T)=\zeta(T) .
$$

Proof: Using induction on (5.1), it is immediate that $M(T) \leq \zeta(T)$. Suppose now that $P_{1}, \ldots, P_{\zeta(T)}$ a set of path cover of $T$. Given a real $\theta$ as an eigenvalue of $A_{\ell} \in \mathcal{H}\left(P_{\ell}\right)$, for all $\ell$, the direct sum $A_{1} \oplus \cdots \oplus A_{\zeta(T)}$ has at least $\zeta(T)$ eigenvalues equal to $\theta$. Therefore $M(T) \geq \zeta(T)$.

Let $q(T)$ be the number of distinct eigenvalues of a Hermitian matrix whose graph is the tree $T$, and $d(T)$ the number of vertices in a longest path of $T$. Johnson and Leal Duarte [18] consider an analog of the well known result for matchings polynomial due to Godsil [8].

Corollary $5.3([8,18])$.

$$
q(T) \geq d(T) .
$$

Proof: From (5.1), for any path,

$$
2 \sum_{\theta}\left(m_{A(T)}(\theta)-1\right) \leq 2 \sum_{\theta} m_{A(T \backslash P)}(\theta) \leq 2|V(T \backslash P)|,
$$

where de sums are over all distinct eigenvalues of $A(T)$. Hence

$$
n-q(T) \leq n-V(P),
$$

for any path, i.e., $q(T) \geq d(T)$.

\section{The Parter-Wiener Theorem}

As we noticed, any weighted tree has at least one essential vertex. In a very interesting work, Parter [21] proved that if $m_{A(T)}(\theta) \geq 2$, then there is a vertex $i$ such that $m_{A(T \backslash i)}(\theta) \geq 3$. In particular, if $m_{A(T)}(\theta)=2$, then $i$ is positive. Later, Wiener [23] proved that if $m_{A(T)}(\theta) \geq 2$, then there is a positive vertex in $T$. If we join these consequences we get the so-called Parter-Wiener Theorem (cf. [15]). 
Theorem 6.1 (Parter-Wiener Theorem). Let $T$ be a tree and suppose that $A \in \mathcal{H}(T)$ such that $m_{A(T)}(\theta) \geq 2$. Then, there is a $\theta$-positive vertex $i$ of $T$ and $\theta$ occurs as an eigenvalue in the direct summands of $A(T)$ which corresponds to at least three branches of $T$ at $i$.

Recently, Johnson, Leal Duarte and Saiago considered the following generalization of the Parter-Wiener Theorem describing also how to choose the positive vertex.

Theorem $6.2([15])$. Let $T$ be a tree and $A \in \mathcal{H}(T)$. If $\theta$ is an eigenvalue of $A(T)$ and there is vertex of $T, j$, such that $m_{A(T \backslash j)}(\theta) \neq 0$, then

(a) there is a $\theta$-positive vertex $i$ of $T$;

(b) if $m_{A(T \backslash i)}(\theta) \geq 2$, then $i$ may be chosen so that $\operatorname{deg}(i) \geq 3$ and so that there are at least three components $T_{1}, T_{2}$ and $T_{3}$ of $T \backslash i$ such that $m_{A\left(T_{\ell}\right)}(\theta) \geq 1$, for $\ell=1,2,3$;

(c) if $m_{A(T \backslash i)}(\theta)=2$, then $i$ may be chosen so that $\operatorname{deg}(i) \geq 2$ and so that there are two components $T_{1}$ and $T_{2}$ of $T \backslash i$ such that $m_{A\left(T_{\ell}\right)}(\theta)=1$, for $\ell=1,2$.

In this section we consider a different approach to the existence of the positive vertex in the tree, based on the tool developed before. The proof shows how to choose in the tree such vertex. But first, we state an important lemma.

Lemma 6.3. Let $T$ be (weighted) tree and $i$ a non-essential vertex in $T$. Then $i$ is positive if and only if there exists $j \sim i$ essential in $T \backslash i$.

Proof: Suppose $i$ is $\theta$-positive and $m_{A(T \backslash i j)}(\theta)>m_{A(T)}(\theta)$, for all $j \sim i$. Then (3.2) leads to a contradiction. Conversely, by a remark of the last section, the path $i j$, for any $j$, is not essential and, consequently, $m_{A(T \backslash i j)}(\theta) \geq m_{A(T)}(\theta)$. Suppose that $j \sim i$ is essential in $T \backslash i$. Then $m_{A(T \backslash i j)}(\theta)=m_{A(T \backslash i)}(\theta)-1$. Hence $i$ is positive.

Theorem 6.4. Let $T$ be a tree and $A \in \mathcal{H}(T)$. If there is a vertex of $T$, say $j$, and a real number $\theta$ such that $m_{A(T)}(\theta) \neq 0$ and $m_{A(T \backslash j)}(\theta) \neq 0$, then there is at least one $\theta$-positive vertex in $T$.

Proof: From (4.3), $\theta$ is a zero of $\varphi\left(T \backslash P_{j k}, \lambda\right)$, for each $k$. Let $S$ be the a subtree of $T$ which is inclusion-minimal subject to the condition $m_{A(S)}(\theta)>$ 0 , and let $i$ be the vertex of $T$ such that $S$ is a component of $T \backslash i$. Let $k \sim i$ 
be a (unique) vertex of $S$ and let $e=(i, k)$. Then $T \backslash e$ has exactly two components, one of which is $S$ and the other we call $R$.

For any component $S^{\prime}$ of $S \backslash k$, we know that $m_{A\left(S^{\prime}\right)}(\theta)=0$, and by Lemma 3.1 we have $m_{A(S \backslash k)}(\theta)=0$. Thus $m_{A(S)}(\theta)=1$. On the other hand, we have

$$
m_{A(T \backslash i)}(\theta)=1+m_{A(R \backslash i)}(\theta) \quad \text { and } \quad m_{A(T \backslash i k)}(\theta)=m_{A(R \backslash i)}(\theta),
$$

i.e., $k$ is essential in $T \backslash i$. Hence, by the Lemma $6.3, \theta$-positive vertex in $T$.

Consider the matrix

$$
A=\left(\begin{array}{cccccccc}
3 & -2 i & 0 & 0 & 0 & 0 & 0 & 0 \\
2 i & 3 & 1+i & 0 & 0 & 0 & 0 & 0 \\
0 & 1-i & 40 & 2 & 0 & 2 & 0 & 0 \\
0 & 0 & 2 & 1 / 2 & 1 / 2 & 0 & 0 & 0 \\
0 & 0 & 0 & 1 / 2 & 1 / 2 & 0 & 0 & 0 \\
0 & 0 & 2 & 0 & 0 & 1 & \sqrt{2} & 0 \\
0 & 0 & 0 & 0 & 0 & \sqrt{2} & -1 & 1 \\
0 & 0 & 0 & 0 & 0 & 0 & 1 & 0
\end{array}\right) .
$$

The graph of $A$ is given by the tree

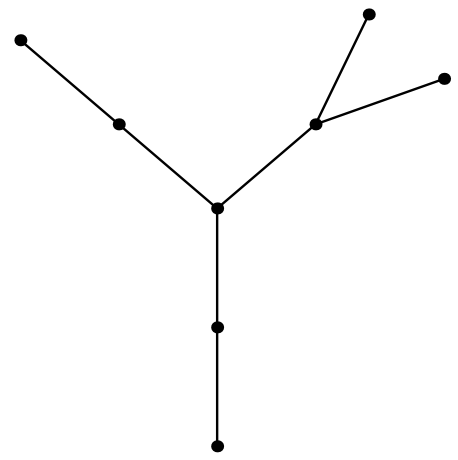

FIG. 1

Notice that 1 is an eigenvalue of $A$ with multiplicity 2 and the vertex 3 is 1-positive.

We know that a tridiagonal matrix has only simple eigenvalues. The next result strengthens this statement. 
Corollary 6.5. Let $T$ be a tree and $A \in \mathcal{H}(T)$. If there exists a $\theta$-essential path $P_{i j}$, for all $j$ in $V(T)$, then $m_{A(T)}(\theta)=1$ and $m_{A(T \backslash k)}(\theta)=0$, for all $k$ in $V(T)$.

Proof: Note the hypothesis implies that all vertices in $T$ are essential. Therefore $m_{A(T \backslash k)}(\theta)=0$, for all $k$ in $V(T)$.

\section{Other consequences}

Finally, using some results on the matchings polynomials, due essentially to Lovász and Plummer [19] and Godsil [8, 9], we can derive some important consequences on the multiplicity of an eigenvalue of weighted trees.

Lemma 7.1. Let $T$ be a weighted tree. If $i$ and $j$ are essential vertices in $T$ and $j$ is not essential in $T \backslash i$, then $P_{i j}$ is essential.

Proof: If $P_{i j}$ is non-essential, by the Theorem 4.4, the multiplicity of $\theta$ as a zero of

$$
\varphi(T \backslash i, \lambda) \varphi(T \backslash j, \lambda)-\varphi(T \backslash i j, \lambda) \varphi(T, \lambda)
$$

is at least $2 m_{A(T)}(\theta)$. Since

$$
m_{A(T \backslash i j)}(\theta) \geq m_{A(T \backslash i)}(\theta)=m_{A(T)}(\theta)-1,
$$

the multiplicity of $\theta$ as a zero of $\varphi(T \backslash i j, \lambda) \varphi(T, \lambda)$ is at least $2 m_{A(T)}(\theta)-1$, and, consequently, the same conclusion applies to $\varphi(T \backslash i, \lambda) \varphi(T \backslash j, \lambda)$. Thus $i$ and $j$ can not both be essential.

We may conclude all paths starting in a vertex in $T$ be essential is equivalent to all vertices in $T$ be essential.

Letting $i$ be a $\theta$-essential vertex in $T$, consider the non-essential path $P$ of minimum length starting in $i$, and let $j$ be the end-vertex. Denote by $P^{\prime}$ the path $P \backslash j$. Then $P^{\prime}$ is essential in $T$ and by interlacing, $m_{A\left(T \backslash P^{\prime}\right)}(\theta)=$ $m_{A(T)}(\theta)$, and $j$ is positive.

Theorem 7.2. Let $T$ be a tree and $A \in \mathcal{H}(T)$. Suppose that $\theta$ is an eigenvalue of $A$ and let $k$ be a non-essential vertex in $T$. Then $i$ is essential in $T$ if and only if it is essential in $T \backslash k$.

Proof: First assume $i$ is essential in $T$. Suppose that $k$ is positive in $T$. Then $m_{A(T \backslash i k)}(\theta)=m_{A(T)}(\theta)$ by interlacing which means that $i$ is essential in $T \backslash k$. Suppose now that $k$ is neutral in $T$. If $i$ is non-essential in $T \backslash k$, then by Theorem 4.4, the multiplicity of $\theta$ as a zero of $\varphi(T \backslash i, \lambda) \varphi(T \backslash k, \lambda)$ is at least $2 m_{A(T)}(\theta)$, which is impossible. 
Similarly, if $i$ is essential in $T \backslash k$, then $i$ is essential in $T$.

Notice that by the this theorem and Lemma 6.3 a positive vertex in a tree has an essential adjacent vertex in the same tree.

If $i$ is a non-essential vertex but it is adjacent to an essential one $j$, then $j$ is essential in $T \backslash i$, by the last theorem. But this implies that $i$ is positive, by the Lemma 6.3. We may state the following result.

Corollary 7.3. A non-essential vertex adjacent to an essential one is positive.

Theorem 7.4. Let $T$ be a tree and $A \in \mathcal{H}(T)$. Let $\theta$ is an eigenvalue of $A$ and $k$ be a positive vertex in $T$. Then

(a) if $i$ is positive in $T$ then it is essential or positive in $T \backslash k$;

(b) if $i$ is neutral in $T$ then it is essential or neutral in $T \backslash k$.

Proof: Suppose that $i$ is positive in $T$ and it is neutral in $T \backslash k$. Once again, by Theorem 4.4, the multiplicity of $\theta$ has a zero of

$$
\varphi(T \backslash i, \lambda) \varphi(T \backslash k, \lambda)-\varphi(T \backslash i k, \lambda) \varphi(T, \lambda)
$$

must be even, in this case at least $2 m_{A(T)}(\theta)+2$. But $m_{A(T \backslash i k)}(\theta)=m_{A(T)}(\theta)+$ 1 , which is a contradiction.

Similarly we prove (b).

\section{References}

[1] F.T. Boesch, S. Chen, J.A.M. McHugh, On the covering the points of a graph with disjoint paths, Graph and Combinatorics, Lect. Notes Math. 406 (1974) 201-212.

[2] T.S. Chihara, An introduction to orthogonal polynomials, Gordon and Breach, New York, 1978.

[3] D.M. Cvetković, M. Doob, H. Sachs, Spectra of graphs, Academic Press, New York, 1979.

[4] E.J. Farrell, Decompositions os some classes of trees into node disjoint paths, Caribb. J. Math. 6 (1987) 35-45.

[5] W. Ferguson, The construction of Jacobi and periodic Jacobi matrices with prescribed spectra, Math. Comp. 35 (1980) 1203-1220.

[6] J. Genin, J.S. Maybee, Mechanical vibration trees, J. Math. Anal. Appl. 45 (1974) 746-763.

[7] C.D. Godsil, Spectra of trees, Ann. Discrete Math. 20 (1984) 151-159.

[8] C.D. Godsil, Algebraic Combinatorics, Chapman and Hall, New York and London, 1993.

[9] C.D. Godsil, Algebraic matching theory, Electron. J. Combin. 2 (1995) Research Paper 8.

[10] F. Harary, The determinant of the adjacency matrix of a graph, SIAM Rev. 4 (1962) 202-210.

[11] O.L. Heilmann, E.H. Lieb, Theory of monomer-dimer systems, Commun. Math. Phys. 25 (1972) 190-232.

[12] R.A. Horn, C.R. Johnson, Matrix Analysis, Cambridge University Press, New York, 1985.

[13] C.R. Johnson, A. Leal Duarte, The maximum multiplicity of an eigenvalue in a matrix whose graph is a tree, Linear and Multilinear Algebra 46 (1999) 139-144. 
[14] C. R. Johnson, A. Leal Duarte, On the possible multiplicities of the eigenvalues of an Hermitian matrix whose graph is a given tree, Linear Algebra Appl., 348 (2002) 7-21.

[15] C. R. Johnson, A. Leal Duarte, C. M. Saiago, The Parter-Wiener theorem: refinement and generalization, SIAM J. Matrix Anal. Appl. 25 (2003) 352-361.

[16] C. R. Johnson, A. Leal Duarte, C. M. Saiago, B. D. Sutton, A. J. Witt, On the relative position of multiple eigenvalues in the spectrum of an Hermitian matrix with a given graph, Linear Algebra Appl. 363 (2003) 147-159.

[17] C. R. Johnson, C. M. Saiago, Estimation of the maximum multiplicity of an eigenvalue in terms of the vertex degrees of the graph of a matrix, Electron. J. Linear Algebra 9 (2002) $27-31$.

[18] A. Leal Duarte, C. R. Johnson, On the minimum number of distinct eigenvalues for a symmetric matrix whose graph is a given tree, Math. Inequal. Appl. 5 (2002) 175-180.

[19] L. Lovász, M.D. Plummer, Matching Theory, Annals Discrete Math. 29, North-Holland, Amsterdam, 1986.

[20] O. Ore, Arc coverings of graphs, Ann. Mat. Pura Appl. 55 (1961) 315-332.

[21] S. Parter, On the eigenvalues and eigenvectors of a class matrices, J. Soc. Indust. Appl. Math. 8 (1960) 376-388.

[22] P.J. Slater, Path coverings of the vertices of a tree, Discrete Math. 25 (1979) 65-74.

[23] G. Wiener, Spectral multiplicity and splitting results for a class of qualitative matrices, Linear Algebra Appl. 61 (1984) 1529.

C.M. DA FONSECA

Departamento de Matemática, Universidade de Coimbra, 3001 - 454 Coimbra, Portugal

E-mail address: cmf@mat.uc.pt 